\title{
FUEL PERFORMANCE CODE COSMOS FOR ANALYSIS OF LWR $\mathrm{UO}_{2}$ AND MOX FUEL
}

\author{
BYUNG-HO LEE ${ }^{1 *}$, YANG-HYUN KOO ${ }^{1}$, JAE-YONG OH ${ }^{1}$, JIN-SIK CHEON ${ }^{1}$, \\ YOUNG-WOOK TAHK ${ }^{1}$, and DONG-SEONG SOHN ${ }^{2}$ \\ ${ }^{1}$ Korea Atomic Energy Research Institute \\ 989-111 Daedeok-daero, Yuseong-gu Daejeon, 305-353, KOREA \\ ${ }^{2}$ UNIST \\ 100 Banyeon-ri, Eonyan-eup, Ulju-gun, Ulsan, 689-798, KOREA \\ ${ }^{*}$ Corresponding author. E-mail : bholee@ kaeri.re.kr
}

Received November 08, 2011

The paper briefs a fuel performance code, COSMOS, which can be utilized for an analysis of the thermal behavior and fission gas release of fuel, up to a high burnup. Of particular concern are the models for the fuel thermal conductivity, the fission gas release, and the cladding corrosion and creep in $\mathrm{UO}_{2}$ fuel. In addition, the code was developed so as to consider the inhomogeneity of MOX fuel, which requires restructuring the thermal conductivity and fission gas release models. These improvements enhanced COSMOS's precision for predicting the in-pile behavior of MOX fuel. The COSMOS code also extends its applicability to the instrumented fuel test in a research reactor. The various in-pile test results were analyzed and compared with the code's prediction. The database consists of the $\mathrm{UO}_{2}$ irradiation test up to an ultra-high burnup, power ramp test of MOX fuel, and instrumented MOX fuel test in a research reactor after base irradiation in a commercial reactor. The comparisons demonstrated that the COSMOS code predicted the in-pile behaviors well, such as the fuel temperature, rod internal pressure, fission gas release, and cladding properties of $\mathrm{MOX}$ and $\mathrm{UO}_{2}$ fuel. This sufficient accuracy reveals that the COSMOS can be utilized by both fuel vendors for fuel design, and license organizations for an understanding of fuel in-pile behaviors.

KEYWORDS : Performance Code, COSMOS, Fuel, Thermal Conductivity, Fission Gas Release, High Burnup

\section{INTRODUCTION}

Fuel vendors and license authorities are using a fuel performance code for designing and validating fuel rods, the behaviors of which are sophisticated during irradiation. Fuel performance codes for $\mathrm{UO}_{2}$ fuel have mainly been developed on an understanding of thermal behavior, fission gas release, and cladding properties such oxidation and creep. In addition, understanding of the high burnup effect has been improved to extend the licensed burnup. Another important aspect in developing a fuel performance code is its proper capacity for analyzing the high burnup mixed oxide (MOX) fuel, since the treatment of large stockpiles of plutonium can be puzzled out when the utilization of MOX fuels can be expedited in commercial light water reactors. To utilize MOX fuels in commercial reactors, there is a lot of research showing the comparable properties of MOX to that of $\mathrm{UO}_{2}$ fuel. One of the important differences comes from an inhomogeneous microstructure, which is caused by the addition of $\mathrm{PuO}_{2}$ particles in the $\mathrm{UO}_{2}$ matrix, with the result that MOX fuel reveals some peculiarities of thermal conductivity and fission gas release.
To predict the complicated in-pile fuel behaviors of $\mathrm{UO}_{2}$ and MOX up to a high burnup, we have been developing the fuel performance code, COSMOS [1]. COSMOS has already been validated by various fuel test databases and revealed its applicability for fuel vendors and license organizations.

In the present paper, COSMOS significant models are summarized. Then, several different in-pile cases are demonstrated for its validation.

\section{FEATURES OF COSMOS CODE}

COSMOS has been developed for the analysis of $\mathrm{UO}_{2}$ and MOX fuel during steady-state and transient operating conditions. The main purpose of COSMOS is to calculate the temperature distribution in the fuel and cladding, as well as fission gas release from the fuel.

Of additional particular concern is the applicability of the COSMOS code for estimating the in-pile behavior of MOX fuel since it is different from typical $\mathrm{UO}_{2}$ fuel in that it contains up to 10 weight $\%$ of plutonium from the 
beginning of irradiation. Due to a difference in the microstructure arising from the addition of plutonium, the following features were implemented in COSMOS when analyzing MOX fuel with the performance models for $\mathrm{UO}_{2}$ fuel: 1) a change in the thermo-mechanical properties, such as the thermal conductivity and thermal expansion coefficient, 2) a change in the radial power depression in a fuel rod as a function of the plutonium fissile content, and 3) a change in the mechanism of the fission gas release resulting from the heterogeneous microstructure of MOX fuel, depending on the manufacturing process.

With due consideration of the above features of MOX fuel and the high burnup characteristics, the main characteristics of the COSMOS code can be summarized as follows.

\subsection{Fission Gas Release Model}

When extending the current LWR fuel burnup, fission gas release is generally considered to be a potential design limitation because of its impact on cladding integrity through an increased internal pressure. In addition, the mechanical restraint caused by PCMI (pellet cladding mechanical interaction), together with high internal pressure, affects the gaseous swelling at the grain boundaries, which is related to the formation of the release path, and hence fission gas release [2,3].

To analyze the fission gas release and gaseous swelling in fuel, a model has been developed that can be applied under both steady-state and transient operating conditions up to a high burnup. A full description can be found in [4], and this paper only gives a brief summary for the fission gas release model.

With an emphasis on the effect of external restraint stress on gas bubbles at the grain boundaries, gaseous swelling, induced by the bubbles located in the grain faces and grain edges, is treated as a function of external stress, which is the sum of internal pressure and external restraint on the rod, caused by PCMI. The external stress, $\sigma$, is considered when the number of gas atoms is calculated in the bubbles of the grain faces or edges. It is assumed that the gas pressure is always balanced by the sum of the lattice surface tension, $\gamma$, and the external stress; that is, the bubbles are always in equilibrium. By applying the ideal gas law, the number of gas atoms, $m$, in a bubble's radius of curvature, $\rho$, required for mechanical stability is given as

$$
m=f(\rho, \theta, r)\left(\frac{2 \gamma}{\rho}+\sigma\right),
$$

where $\theta$ is the semi-dihedral angle defining the shape of a gas bubble, and $r$ a radius of curvature of a circular grain face. The concentration of grain face bubbles is given as a function of temperature.

The model was compared with the data obtained from commercial reactors, Ris $\phi$-III Project, isothermal irradiation, and post irradiation annealing experiments (See Fig. 1). It is shown that the model predicts well the fractional fission gas release.

These comparisons with the in-pile data suggest that the present model can be used for the analysis of fission gas release in high burnup fuel, where strong external restraint stress may develop due to pellet-cladding interaction.

In the case of MOX fuel, some heterogeneity exists in the microstructure depending on the manufacturing method, due to the incomplete mixing of $\mathrm{PuO}_{2}$ powder with $\mathrm{UO}_{2}$ powder. There is some controversy over whether or not fission gas release is enhanced in MOX fuel compared with $\mathrm{UO}_{2}$ fuel under similar operating conditions. Recent experiments showed that there appeared to be little effect of the microstructure in currently produced fuel. In addition, high gas release in MOX fuel is being attributed to higher operating powers in this fuel later in its life, because the reactivity falls more slowly with burnup than that of $\mathrm{UO}_{2}$ fuel. However, it is necessary to have the ability to consider the effect of heterogeneity to analyze MOX fuel.

To analyze the effect of microscopic heterogeneity on fission gas release, a spherical model has been developed using the assumption that Pu-rich particles are distributed uniformly in a $\mathrm{UO}_{2}$ matrix. The fuel pellet is assumed to consist of equivalent spherical particles with a diameter of $\mathrm{D}_{\mathrm{eq}}=\mathrm{D}_{\mathrm{agg}}+2 \mathrm{~L}_{\mathrm{rec}}$, surrounded by the $\mathrm{UO}_{2}$ matrix containing a Pu-rich particle, where $\mathrm{L}_{\mathrm{rec}}$ is the recoil length of the fission products (see Fig. 2). The recoil length is assumed to be $6 \mu \mathrm{m}$. The diameter of an equivalent cell is then defined as follows:

$$
D_{\text {cell }}=D_{\text {agg }} / \sqrt[3]{\frac{e_{p}-e_{m}}{e_{a}-e_{m}}}
$$

where $e_{p}$ is the average Pu content of the pellet, $e_{a}$ is the $\mathrm{Pu}$ content of the $\mathrm{Pu}$-rich particle, $\mathrm{e}_{\mathrm{m}}$ is the $\mathrm{Pu}$-content in the $\mathrm{UO}_{2}$ matrix, and $\mathrm{D}_{\text {agg }}$ is the average size of the $\mathrm{Pu}$-rich particle.

The fission rates in the equivalent cell and matrix are calculated considering the effective enrichments in each zone. If the fuel parameters are such that $\mathrm{D}_{\mathrm{eq}}$ is larger than $\mathrm{D}_{\text {cell }}$, the fuel can be considered homogeneous.

The equivalent $\mathrm{Pu}$ content of the cell is calculated as follows:

$$
e_{e q}=e_{m}+\left(\frac{D_{a g g}}{D_{e q}}\right)^{3} \cdot\left(e_{a}-e_{m}\right)
$$

The fission rates in the equivalent cell and $\mathrm{UO}_{2}$ matrix are given below, where the average fission rate $\mathrm{F}_{\mathrm{av}}$ can be obtained from the average power density:

$$
F_{e q}=\left(\frac{e_{e q}}{e_{p}}\right) \cdot F_{a v} \text { and } F_{m}=\left(\frac{e_{m}}{e_{p}}\right) \cdot F_{a v}
$$


The average fission rates in each region are incorporated into the fission gas release model for $\mathrm{UO}_{2}$ fuel [2], and are used to calculate the gas release in their respective regions.

This model, considering the inhomogeneity of MOX fuel, has been proven by using the data from international programs [6].

\subsection{Thermal Conductivity Model}

The thermal conductivity recommended by Halden [7] was adopted for $\mathrm{UO}_{2}$ fuel, whereas a general thermal conductivity model applicable for a two-phase material was developed for MOX fuel [8].

The thermal conductivity of MOX fuel can be
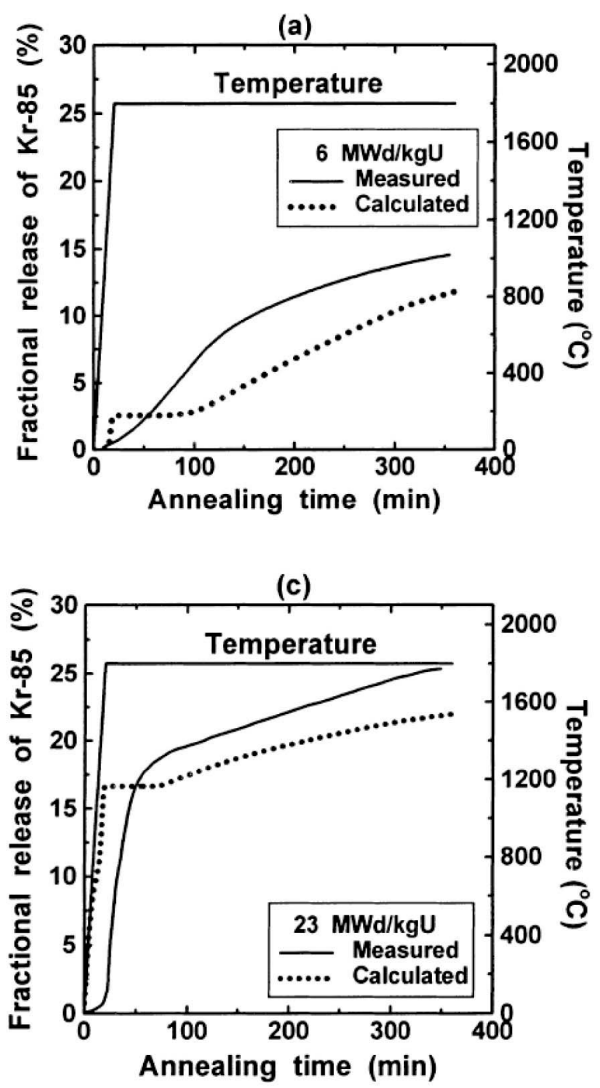

(b)

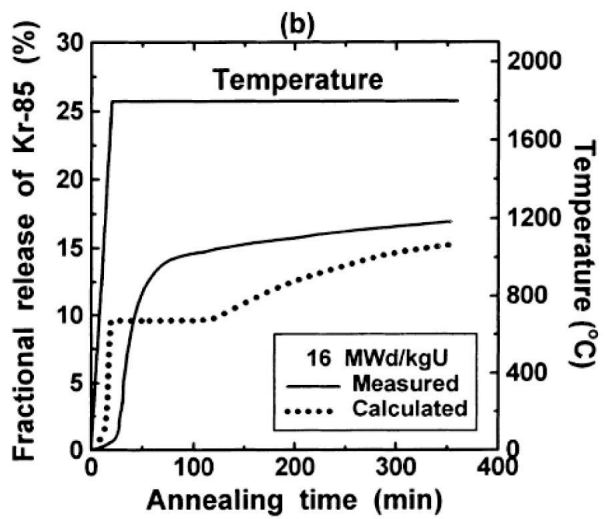

(d)

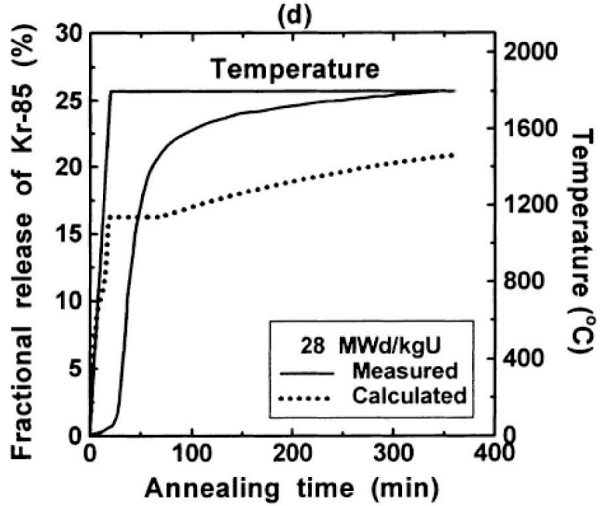

Fig. 1. Fractional Release of $\mathrm{Kr}-85$ during Temperature Ramp and Isothermal Annealing at $1800^{\circ} \mathrm{C}$ [5].

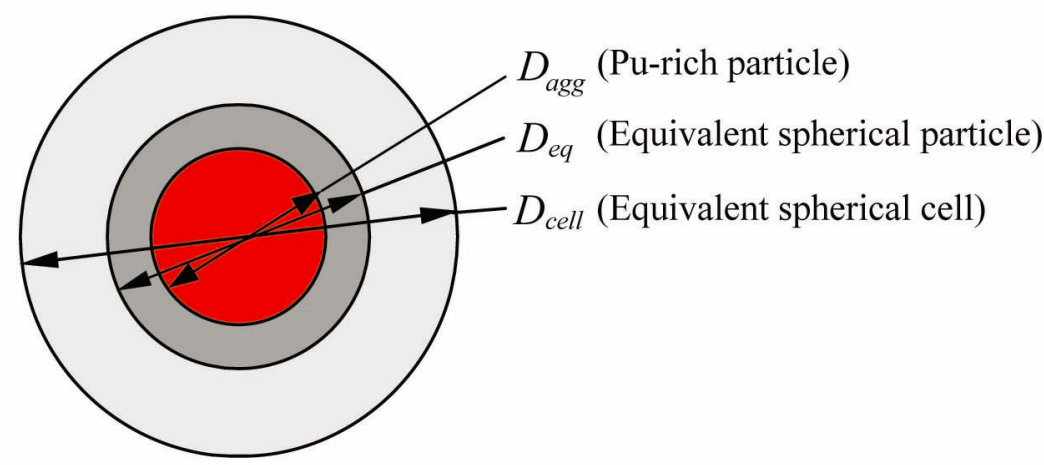

Fig. 2. An Equivalent Spherical Cell for a Pu-rich Particle 
estimated from a combination of the thermal conductivities for matrix and plutonium-rich agglomerates:

$$
k_{\text {MOX }}=k_{\text {matrix }} \cdot\left\{\begin{aligned}
& 1-a \cdot P_{P u R}{ }^{\frac{2}{3}} \\
&\left.\cdot\left[1-1 /\left(1+\frac{1}{a} \cdot P_{P u R} \frac{1}{3} \cdot\left(\frac{k_{\text {matrix }}}{k_{P U R}}-1\right)\right)\right]\right\}
\end{aligned}\right.
$$

where

$k_{M O X}=$ the integral thermal conductivity of a heterogeneous MOX fuel,

$k_{\text {matrix }}=$ the thermal conductivity of a matrix in which a small fraction of the plutonium content is included

$k_{P U R}=$ the thermal conductivity of plutonium-rich particles

$P_{P u R}=$ the volumetric fraction of plutonium-rich particles, and

$a \quad=$ the anisotropy factor ( $a=1$ means isotropic pore distribution).

The thermal conductivity of the matrix is derived from a combination of Halden's model [7] and some relevant information on MOX fuel. The stoichiometry effect on the thermal conductivity is assumed to be negligible for the analysis of the in-pile thermal behavior of MOX fuels.

The resistance of phonon-impurity increases with plutonium embedded in the matrix, which is assumed to be proportional to the plutonium content. On the other hand, the lattice resistivity theory was applied to estimate the resistance by phonon-phonon interaction.

Philliponneau's model [9] is applied for the local thermal conductivity of plutonium-rich particles.

The thermal conductivity of homogeneous MOX fuel ranges from 85 to $92 \%$ of that of $\mathrm{UO}_{2}$ at the beginningof-its-life (BOL), whereas with inhomogeneous MOX, the thermal conductivity decreases by 4 to $8 \%$ when compared to $\mathrm{UO}_{2}$ fuel at the BOL.

This estimated reduction is comparable to ENIGMA [10] and Siemen's [11] MOX thermal conductivities, which are shown to be $8 \%$ less than that of the standard $\mathrm{UO}_{2}$ fuel and a relative decrease of $4 \sim 5 \%$, respectively. However, these are slightly more conservative than BelgoNucleaire's results [12], which showed a $4 \%$ reduction of the thermal conductivity for $10 \% \mathrm{Pu} /(\mathrm{Pu}+\mathrm{U})$ fuel.

The developed thermal conductivity model was verified by various tests, which have measured the fuel centerline temperature by installation of a thermocouple or expansion thermometer in the fuel center. The tests cover homogeneous and inhomogeneous MOX fuel irradiated up to a high burnup, with or without a significant fission gas release.

An example is demonstrated in Fig. 3. The fuel centerline temperature was measured using an expansion thermometer and power corresponding to the average linear heating rate of the rod. The calculated centerline temperature shows a very good agreement with the measured temperature. The fuel temperature increases linearly with the linear heating rate. This calculation suggests that the developed thermal conductivity model predicts well the BOL thermal behavior in MOX fuel.

\subsection{Corrosion and Creep Model for Cladding}

The cladding of zirconium alloy is the first safety barrier, and its performance plays an important role in achieving a very high burnup. In addition, an accurate assessment for the cladding behaviors is a prerequisite to obtain the precise thermal behavior of fuel rods. In these regards, the corrosion and creep models were improved and validated using the database.

The improved corrosion model takes into account the tin content, water chemistry, and heat treatment, such as the accumulated annealing parameter. In particular, the model assumes that the sub-cooled void present in the coolant is a prerequisite for the detrimental water chemistry effect to accelerate corrosion in a cladding.

The developed model was evaluated to see whether the corrosion model is adequate for predicting the corrosion behavior of 40 different fuel rods [13]. Fig. 4 shows a comparison between the measured and predicted oxide thickness.

If it is assumed that the complicated corrosion environments and cladding manufacturing data are different for more than 10 years, COSMOS predictions indicate that the corrosion model is suitable for a corrosion estimation in various irradiation environments. The model was also validated using the database obtained from high-burnup $\mathrm{UO}_{2}$ and MOX fuels in commercial reactors. A comparison of the predicted oxide thickness with the measured data confirms that the corrosion model in COSMOS is proper for analyzing the cladding oxidation of the fuel, up to a high burnup.

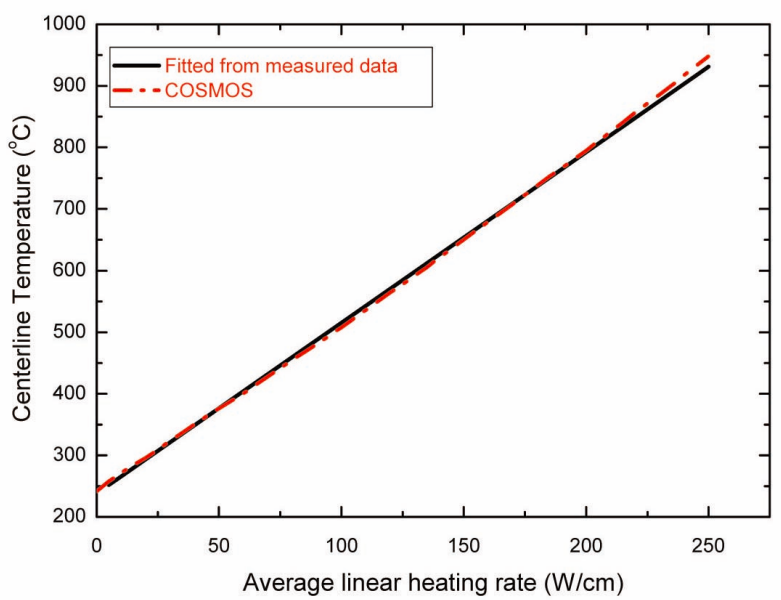

Fig. 3. Comparison of Measured Temperatures using Expansion Thermometer with COSMOS Prediction for Homogeneous MOX Fuel. 
The creep model [14] was also improved to take into account heat treatment for cladding fabrication. Based on the experimental results in which the creep strain rate is highest for stress relief annealed cladding (SRA) and lowest for recrystallized annealed cladding (RXA), the annealing factor was introduced and derived using iterative calculations with a trial formulation until the best predictions for all the rods were obtained. As shown in Fig. 5, there are no discernible differences between the measured and calculated creep behaviors.

\subsection{The Other COSMOS Features}

By mainly focusing on the thermal conductivity and fission gas release in MOX fuel, the other characteristics of COSMOS are as follows:

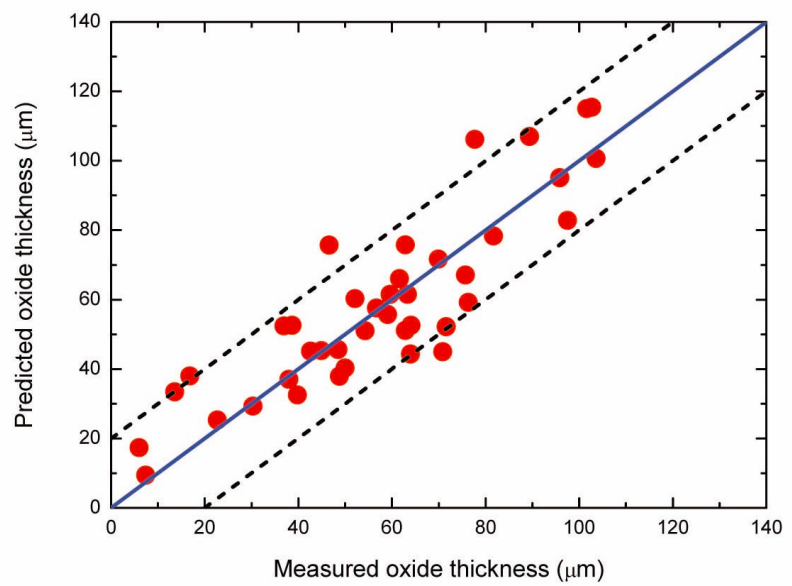

Fig. 4. Comparison between the Measured and Predicted Oxide Thickness

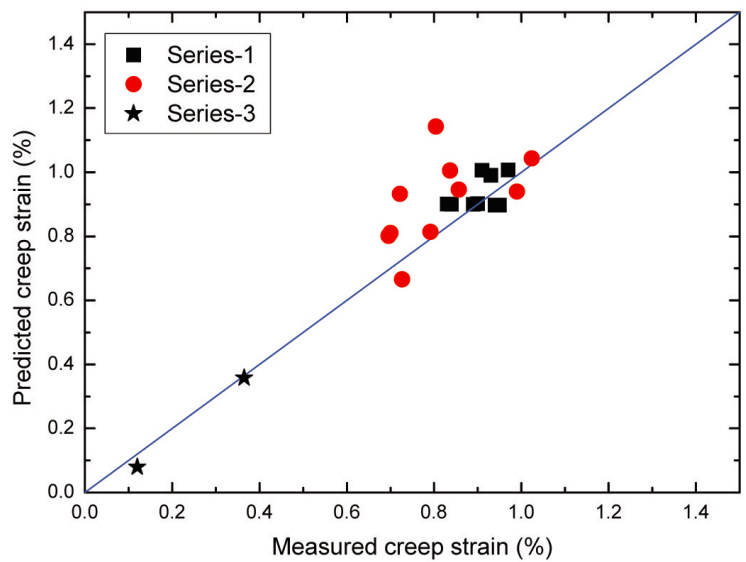

Fig. 5. Comparison of the Predicted and Measured Creep Strains
- COSMOS has been improved with a Graphic User Interface (GUI) based on the Windows operating system. Since the text structure of the inputs and outputs was inconvenient, COSMOS was implemented with a GUI system for creating those files.

- In addition, the execution of COSMOS can be made by an input dialog offering various options in a window for calculation. Although the menu system is very convenient for controlling one input file, it is not suitable for treating many input files at once. Hence, COSMOS also provides a batch interface for handling many input files simultaneously. The batch interface reads the batch command file, which contains information on the input file names and control parameters.

- For a clear and intuitive understanding of the results, COSMOS generates not only output files based on the text format, but also graphic results. Identical graphs are displayed on the screen and in a postscript file.

- An important advantage of COSMOS is that it can analyze fuel segments re-fabricated from base-irradiated fuel rods. This makes it possible to utilize a database obtained from international projects such as HALDEN and RIS $\phi$, many of which were collected from refabricated fuel segments. In particular, COSMOS has been improved to estimate the fuel centerline temperature of fuel rods equipped with a thermocouple.

- Based on the measured rim characteristics of a high burnup $\mathrm{UO}_{2}$ fuel, the pressure of the rim pores and an additional pellet swelling due to a rim formation have been modeled as a function of temperature, pellet average burnup, and pore radius. This information can be used to analyze fuel behavior under reactivity initiated accident conditions during which the pores with a high pressure may cause a crack propagation along the sub-grain boundaries, resulting in a ventilation of the gas atoms retained in the pores. In addition, thermal conductivity degradation due to a porous rim formation is considered.

\section{VERIFICATION OF COSMOS CODE WITH IN- PILE DATA}

COSMOS has been validated substantially with the MOX database as well as with many other databases for high burnup $\mathrm{UO}_{2}$ fuels. The following exhibits validations using various in-pile databases.

\subsection{Thermal Behaviour of $\mathrm{UO}_{2}$ Fuel Up to Ultra-high Burnup}

It is essential that a fuel performance code can predict the in-pile fuel thermal behavior as accurately as possible for a proper assessment of all other in-pile behaviors. In this regard, the first verification demonstrates the prediction 
of thermal behavior by using a well-known database obtained by the IFA-562 test. This test aimed at quantifying the $\mathrm{UO}_{2}$ conductivity degradation up to a very high burnup [15].

The test was performed with six fuel segments, the diameters of which were small enough to achieve a fast burnup accumulation (See Table 1). Validation for one fuel segment was presented in the paper, since all the fuel segments were siblings.

An expansion thermometer at the center hole of the whole fuel stack length measured the fuel centerline temperature, and an accurate determination of the power distribution was achieved with four vanadium neutron detectors. The fuel center temperature remained well below the threshold for a fission gas release, and the burnup increased to about $87 \mathrm{MWd} / \mathrm{kgU}$.

The calculated centerline temperature is consistent with the measured temperature (Fig. 6). The power and temperature decreased with irradiation, due to the depletion of fissile material. These agreements indicate that COSMOS simulates thermal behaviors up to a very high burnup, by considering the fuel geometrical changes and fuel thermal conductivity properly.

Table 1. Main Design Parameters of IFA-562

\begin{tabular}{|c|c|}
\hline FUEL & \\
\hline Weight, $g$ & 110 \\
\hline Density of TD, $\%$ & 94 \\
\hline Enrichment, \% & 13 \\
\hline Grain Size, $\mu \mathrm{m}$ & $10 \sim 20$ \\
\hline Pin length, mm & 442.5 \\
\hline \multicolumn{2}{|l|}{ PELLET GEOMETRY } \\
\hline Length, mm & 7.5 \\
\hline Inner diameter, $\mathrm{mm}$ & 2.0 \\
\hline Outer diameter, $\mathrm{mm}$ & 5.915 \\
\hline Dishing & none \\
\hline \multicolumn{2}{|l|}{ CLADDING } \\
\hline Material & $\mathrm{Zr}-2$ \\
\hline Inner diameter, mm & 6.015 \\
\hline Thickness, mm & 0.50 \\
\hline Filling gas & Helium 10 bar \\
\hline Free volume, $\mathrm{cm}^{3}$ & 4.1 \\
\hline Plenum length, mm & 31.0 \\
\hline
\end{tabular}

\subsection{PRIMO Program}

One MOX fuel rod (Fig. 7) in-pile tested in the framework of PRIMO was adopted for verification [16,17]. The PRIMO program was used in an investigation of MOX fuel to demonstrate the fuel's compatibility in PWRs during steady and ramp irradiation tests.

The fuel rod was manufactured at BelgoNucleaire using a MIMAS (Micronized Master Blend) process. The use of a master blend leads to some plutonium-rich particles in MOX fuels, which can locally reach the $\sim 30 \% \mathrm{PuO}_{2}$ content of the primary blend.

The MOX fuel rod was base irradiated in the BR3 reactor, up to the peak pellet burnup of $38 \mathrm{MWd} / \mathrm{kgHM}$. After the base irradiation, the fuel rod was submitted to a power excursion in the OSIRIS reactor. The fuel rod was preconditioned at a peak LHGR of $189 \mathrm{~W} / \mathrm{cm}$ for 27 hours. The subsequent power excursion reached a terminal power of $395 \mathrm{~W} / \mathrm{cm}$ during 20 hours, with a ramping rate of $77 \mathrm{~W} / \mathrm{cm}$-min. Non-destructive examinations were performed on the base irradiated MOX fuel. The destructive examinations were performed after a ramp test, which revealed a fission gas release of $11.24 \%$ with an Xe-to-Kr ratio of 15.4.

To analyze the MOX fuel behavior by the COSMOS code, a power history (Fig. 8) during the base irradiation was simulated first to obtain the incipient conditions of the ramping test. The power history was combined with the axial power peaking, defined in twelve axial zones. The ramping test was simulated by the power history shown in Fig. 9 with cosine-shaped axial power peaking (Fig. 10).

The gas puncturing data performed after power ramping was compared with the COSMOS prediction.

The COSMOS code analyzed the fission gas release with two options [4]: (1) activating the option for a burst release through micro-cracks occurring during a rapid power transient, and (2) not activating the burst release option. Fig. 11 shows both calculation results. The measured fission gas release is also displayed in the figure. It can be seen that the fission gas release, while considering the burst fission gas release through micro-cracks, agrees well with the measured value. The comparison confirms the assumption that the entire fission gas inventory at the grain boundaries is released instantaneously during rapid power ramping.

\subsection{Instrumented MOX Fuel Test after Base Irradiation}

For verification of the models of thermal conductivity and fission gas release in MOX fuel, a fully detailed analysis is performed for two full length fuel rods during the base irradiation in a commercial reactor, and for four re-instrumented segments during the test irradiation in a test reactor [18].

The four-segment behavior was modeled in two steps. Firstly, the calculation for the base irradiation of 

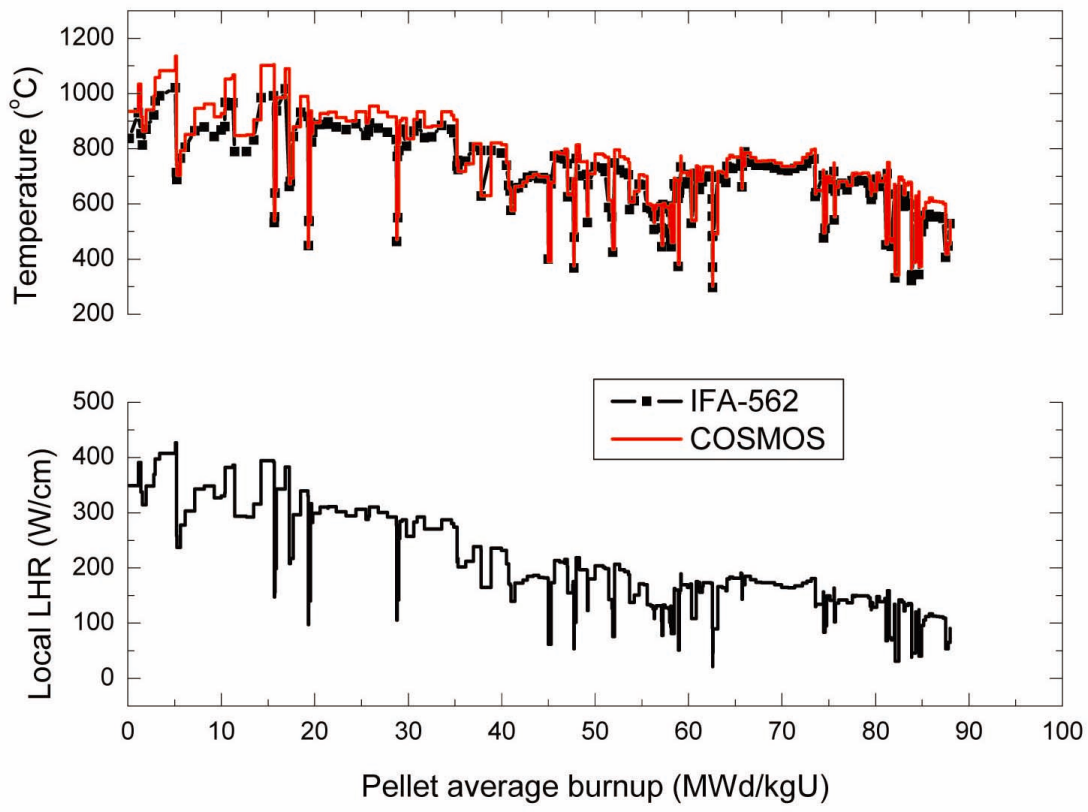

Fig. 6. Fuel Temperature of IFA-562

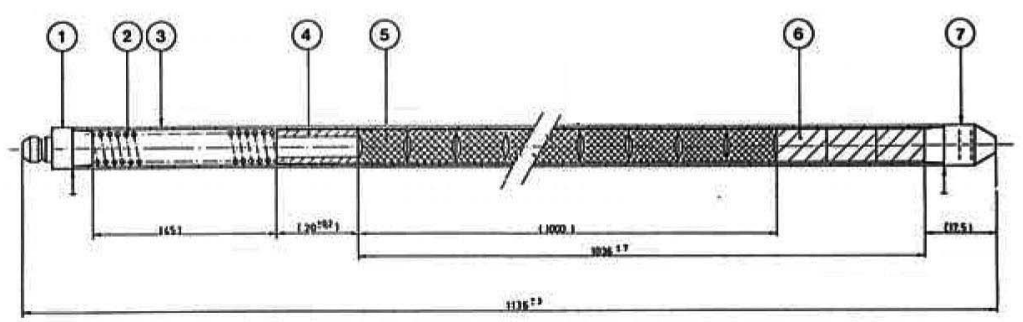

Fig. 7. Schematics of the MOX Fuel from the PRIMO Program: (1) Upper-end Plug (2) Hold Down Spring (3) Cladding Tube (4) Spacer Tube (5) MOX Fuel Pellet (6) $\mathrm{UO}_{2}$ Blanket Pellet (7) Lower-end Plug.

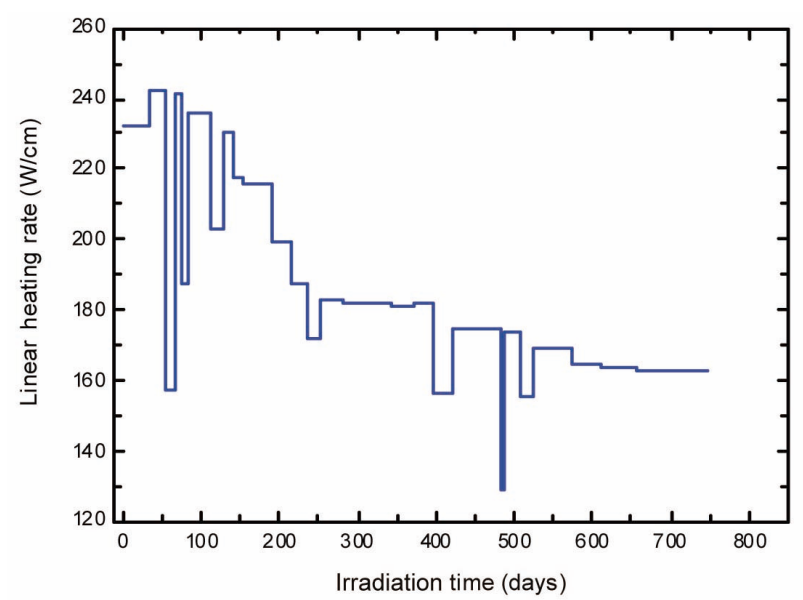

Fig. 8. Heating Rate during Base Irradiation

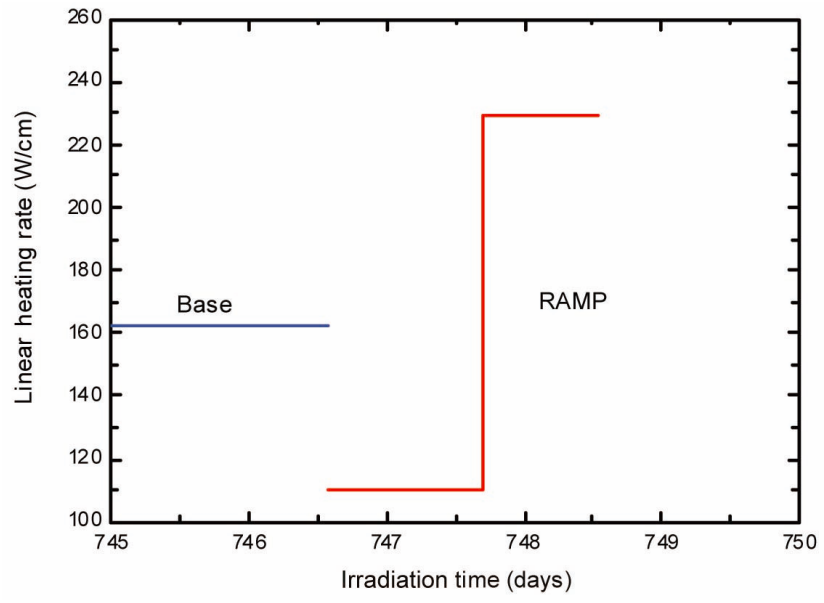

Fig. 9. Linear Heating Rate of the Power Ramp 


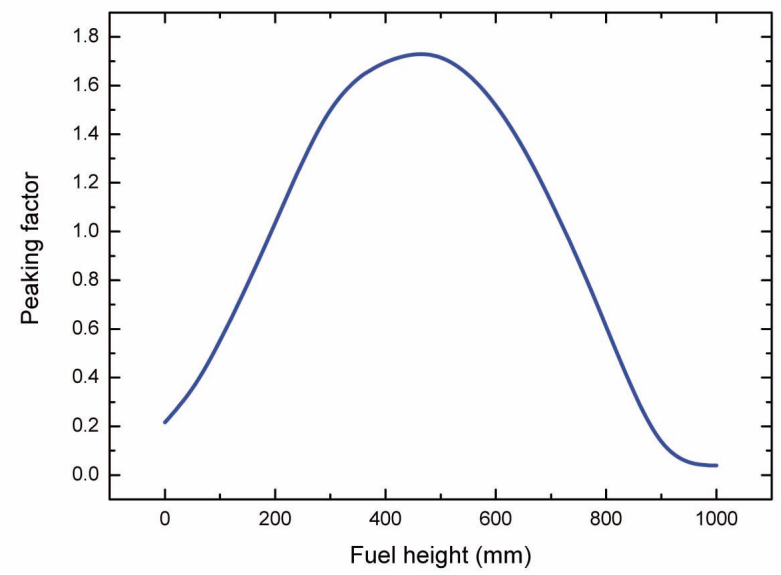

Fig. 10. Peaking Factor in the OSIRIS Ramp

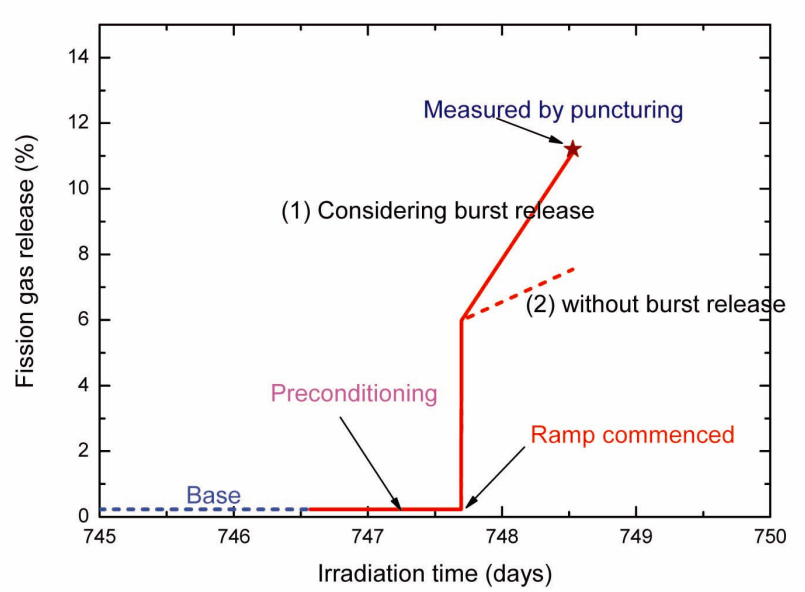

Fig. 11. Comparison between Measured and Calculated FGR at EOL

full size fuel rods, Rod-1 and Rod-2, was fulfilled. The first calculation stored the results of the fission gas atom distribution at the grain interiors, and at the grain boundaries, grain sizes, and some other values. During the second calculations with a new geometry and gas filled content for each segment, the data stored after the base irradiation calculation were read in. These data were used as initial data for the second step calculations for the re-instrumented irradiation test.

During all base irradiation, the predicted central fuel temperature did not exceed Vitanza' threshold temperature in either fuel rod. No restructuring was predicted during the base irradiation, which agreed with the post-irradiation examination. Nevertheless, a small fission gas release was predicted for both fuel rods due to thermal diffusion (see Fig. 12 and Fig. 13).

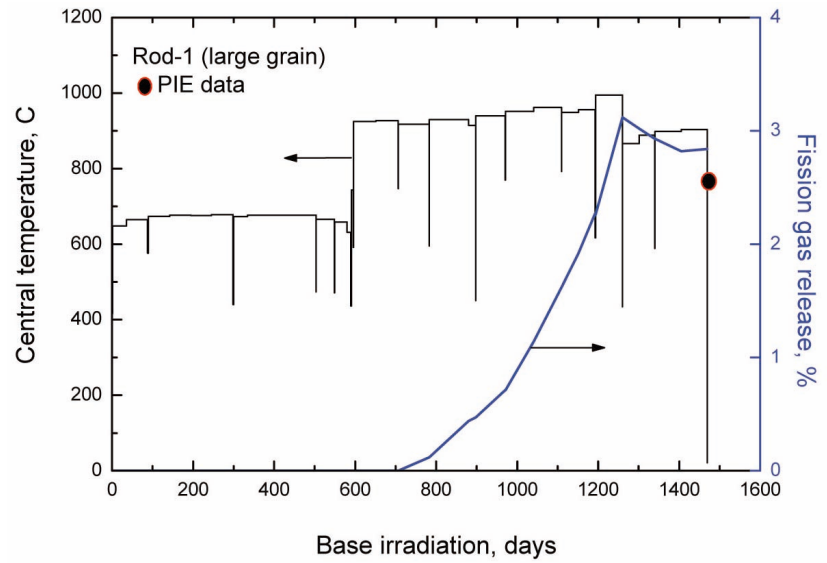

Fig. 12. Fuel Temperature and FGR for Rod-1

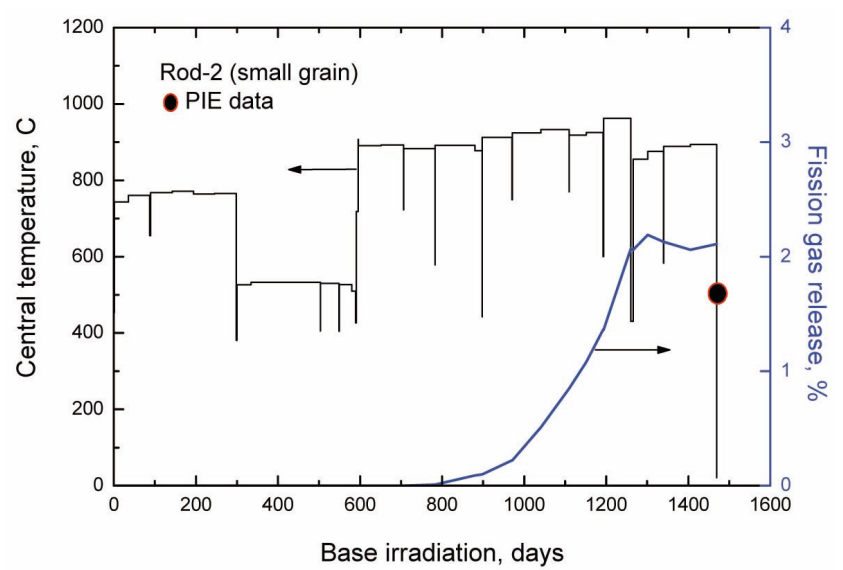

Fig. 13. Fuel Temperature and FGR for Rod-2

The fission gas release for Rod-1 with a large grain size, is higher than for Rod-2 with a small grain size. This can be explained by the fact that burnup for Rod-1 was slightly higher than that for Rod-2, but also by a higher power history in Rod-1.

A re-instrumented irradiation test was performed to investigate the fission gas release by the diffusional process. Segment-1 was extracted from Rod-1, whereas Segment-2 was prepared from Rod-2. Fig. 14 and Fig. 15 contain the comparison of the calculation and experimental data for the re-instrumented irradiation test.

As seen from Fig. 14, the COSMOS code predicts the thermal behaviors for a fuel segment instrumented with a thermocouple very accurately. The comparison reveals that the COSMOS code was implemented with the proper models so as to predict the in-pile fuel behaviors with a very sophisticated and step-wise power history. 


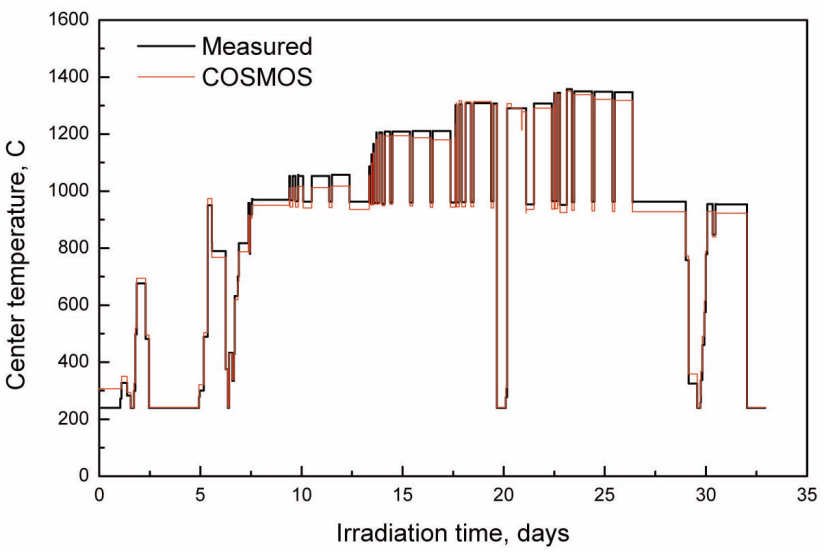

Fig. 14. Comparison of Calculated and Experimental Fuel Temperature for Segment-1

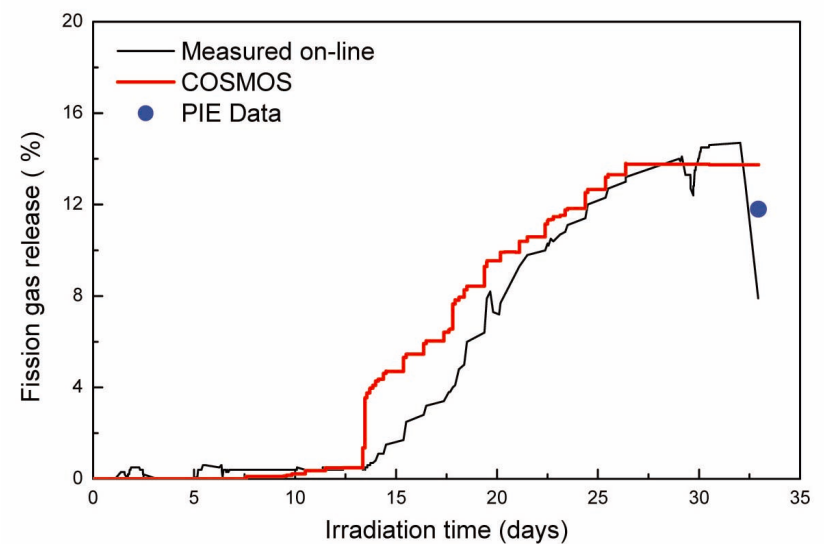

Fig. 15. Comparison of Calculated and Experimental FGRs for Segment-2.
Fig. 15 demonstrates that the COSMOS predicts the kinetics of rod internal pressure, which means COSMOS predicts the fission gas release, as well as the fuel geometrical changes. Finally, the PIE data reveals that COSMOS calculates the amount of fission gas release very precisely.

\section{CONCLUSIONS}

A fuel performance code, COSMOS, has been developed and sustained improvements for the analysis of $\mathrm{UO}_{2}$ and MOX fuel, up to a high burnup. The COSMOS code is equipped with precise models to understand the in-pile fuel behaviors that are required for proper design and license. The models are related to the fission gas release, temperature distribution, and the corrosion and creep of claddings. In addition, the code has all the required userfriendly functions for intuitive awareness after a fuel performance analysis.

This paper demonstrated an analysis by the COSMOS code for $\mathrm{UO}_{2}$ fuel with a very high burnup, the power ramp of MOX fuel, and a re-instrumented MOX fuel after base irradiation in a commercial reactor. These representative demonstrations confirm COSMOS's applicability for analyzing all varieties of irradiation cases for $\mathrm{UO}_{2}$ and MOX fuels, up to a high burnup.

\section{ACKNOWLEDGMENTS}

The authors would like to express their appreciation to the Ministry of Education, Science and Technology (MEST) of the Republic of Korea for the support of this work through the mid- and long-term Nuclear R\&D Project. We also wish to acknowledge the help of EdF and the Halden Reactor Project staffs.

\section{REFERENCES}

[ 1 ] Y.H.Koo, B.H. Lee, D.S. Sohn, "COSMOS: a Computer Code to Analyze LWR $\mathrm{UO}_{2}$ and MOX Fuel up to High Burnup", Annals of Nuclear Energy, 26, 47 (1999).

[2 ] H. Zimmermann, "Investigations on Swelling and Fission Gas Behavior in Uranium Oxide”, J. Nucl. Mater. 75 (1978) 154.

[3] T. Kogai, K. Ito and Y. Iwano, "The Effect of Cladding Restraint on Fission Gas Release Behaviors", J. Nucl. Mater. 158, 64 (1988).

[4] Y.H. Koo, et al "Analysis of Fission gas release and gaseous Swelling in $\mathrm{UO}_{2}$ Fuel under the Effect of External Restraint", J. Nucl. Mater. 28086 (2000).

[ 5 ] K. Une and S. Kashibe, J. Nucl Sci. Tech. 27, 1002 (1990).

[6] Y.H. Koo, B.H. Lee, J.S. Cheon, D.S. Sohn, "Modeling and parametric studies of the effect of inhomogeneity on fission gas release in LWR MOX fuel", Annals of Nuclear Energy, 29, 271 (2002).

[ 7 ] W. Wiesenack, T. Tverberg, "Thermal Performance of High Burnup FueĨ In-pile Temperature Data and Analysis", 2000 International Topical Meeting on LWR Fuel Performance, Park City, Utah, April, 2000.

[ 8 ] H. Kampf and G. Karsten, "Effect of Different Types of Void Volumes on the Radial Temperature Distribution of Fuel Pins", Nucl. Appl. Tech., 9, 288 (1970).

[9] Y. Philipponneau, "Thermal Conductivity of $(\mathrm{U}, \mathrm{Pu}) \mathrm{O}_{2-x}$ Mixed Oxide Fuel”, J. Nucl. Maters., 188, 194 (1992).

[10] G. Gates, et al., "Thermal Performance Modeling with the ENIGMA Code", Proc. of Seminar on Thermal Performance of High Burnup LWR Fuel, France, p.301 (1998).

[11] L. Heins, H. Landskron, "Fuel Rod Design by Statistical Methods for MOX Fuel", Int. Symposium on MOX Fuel Cycle Technologies for Medium and Long-term Deployment, IAEA-SM-358/21, Austria (1999).

[12] M. Lippens, et al., "Comparative Thermal Behavior of MOX Fuel and $\mathrm{UO}_{2}$ Fuels", Proc. of the Seminar on Thermal Performance of High Burnup LWR Fuel, France, p.243 (1998).

[13] B.H. Lee, Y.H. Koo, J.Y. Oh, D.S. Sohn, "Zircaloy-4 
cladding corrosion model covering a wide range of PWR experiences", 378, 127 (2008).

[14] B.H. Lee, Y.H. Koo, Cheon, D.S. Sohn, "Modeling of creep behavior of Zircaloy-4 by considering metallurgical effect", Annals of Nuclear Energy 29, 1 (2002).

[15] Y. Kosaka, “Thermal Conductivity Degradation Analysis of the Ultra High Burnup Experiment (IFA-562)", HWR341, Halden Reactor Project.

[16] L.J. Ott, T. Tverberg, E. Sartori, Annals of Nuclear Energy, 36, 375 (2009).

[17] L.J. Ott, NEA/NSC/DOC (2009)

[18] M. Lipppens, FIGARO International Programme, 2000 